\title{
Detecção de Enterobacterales produtoras de carbapenemases em pacientes colonizados, atendidos em um Hospital Universitário
}

\author{
Detection of Carbapenemase-Producing Enterobacterales in Colonized Patients, Attended \\ at a University Hospital
}

\author{
Detección de Enterobacterales Productores de Carbapenemasas en Pacientes \\ Colonizados, Atendidos en un Hospital Universitario
}

Mariana Magaldi de Souza Lima ${ }^{1}$, Daniela Greco Garcia Fernandes ${ }^{1}$, Elaini Aparecida de Oliveira ${ }^{1}$, Maikeline Paraguassú Pinheiro ${ }^{1}$, Douglas Guedes Ferreira² ${ }^{2}$ Viviane Zahner ${ }^{3}$, Thiago Pavoni Gomes Chagas $^{1}$, Cláudia Rezende Vieira de Mendonça-Souza ${ }^{1 *}$.

\section{RESUMO}

Objetivo: Investigar a produção de carbapenemase entre amostras de Enterobacterales obtidas a partir de culturas de vigilância, de pacientes internados em um hospital universitário no Rio de Janeiro, bem como a distribuição das espécies bacterianas e seus perfis de sensibilidade. Métodos: Os swabs retais foram coletados na admissão dos pacientes e, após, semanalmente, nas unidades: Coronariana, Diálise, Emergência, Hematológica, Pediatria, e de Terapia Intensiva. As amostras obtidas foram submetidas a métodos fenotípicos para detecção de produção de carbapenemases. A identificação bacteriana foi realizada por meio de espectrometria de massas. O perfil de sensibilidade aos antimicrobianos foi determinado por disco-difusão e a detecção dos determinantes genéticos foi realizada por meio da Reação em Cadeia da Polimerase. Resultados: A produção de carbapenemases foi observada em $34,9 \%$ dos isolados. As espécies mais frequentes foram Klebsiella pneumoniae (68,4\%) e Escherichia coli $(15,8 \%)$. Uma elevada taxa de multirresistência (89,5\%) foi observada entre as Enterobacterales produtoras de carbapenemases (EPC). Verificou-se que $81,6 \%$ das amostras apresentavam o gene blakPC e 15,8\%, o blandM. Duas amostras foram concomitantemente positivas para os genes blakpc/blandm. Conclusão: Foi observada uma elevada taxa de colonização por EPC multirresistentes, com predominância de K. pneumoniae carreando blakPC.

Palavras-chave: Bactérias gram-negativas, Microbiota, Controle de infecções.

\begin{abstract}
Objective: To investigate of the production of carbapenemase among Enterobacterales isolates obtained from surveillance cultures from patients attended at a university hospital in Rio de Janeiro, as well as the distribution of bacterial species and their susceptibility patterns. Methods: Rectal swabs were collected at the admission of patients and, afterwards, weekly, in the units: Coronary, Dialysis, Emergency, Hematological, Pediatrics, and Intensive Care. The isolates obtained were submitted to phenotypic methods for the detection of carbapenemase production. Bacterial identification was performed using mass spectrometry. The antimicrobial susceptibility was determined by disk-diffusion and the detection of the genetic determinants was carried out through the Polymerase Chain Reaction. Results: The production of carbapenemases was observed in 34.9\% of the isolates. The most frequent bacterial species were Klebsiella pneumoniae (68.4\%) and Escherichia coli
\end{abstract}

\footnotetext{
1 Universidade Federal Fluminense (UFF), Niterói - RJ. *E-mail: claudia_souza@id.uff.br

2 Hospital Universitário Antônio Pedro (HUAP), Niterói - RJ.

${ }^{3}$ Fundação Oswaldo Cruz (IOC-LEMEF), Rio de Janeiro - RJ
} 
(15.8\%). A high rate of multidrug resistance (89.5\%) was observed among the carbapenemase-producing Enterobacterales (CPE). It was found that $81.6 \%$ of the isolates had the blakPC gene and $15.8 \%$, blandM. None of the isolates presented the blaoxA-48-like gene. Two isolates were concomitantly positive for blakPC/blanDM genes. Conclusion: A high rate of colonization by multidrug-resistant CPE was observed, with a predominance of $K$. pneumoniae carrying blakpc.

Keywords: Gram-negative bacteria, Microbiota, Infection control.

\section{RESUMEN}

Objetivo: Investigar la producción de carbapenemasa entre muestras de Enterobacterales obtenidas de cultivos de vigilancia de pacientes ingresados en un hospital universitario de Río de Janeiro, así como la distribución de especies bacterianas y sus perfiles de sensibilidad. Métodos: Se recogieron hisopos rectales al ingreso de los pacientes y, posteriormente, semanalmente en las unidades: Coronario, Diálisis, Urgencias, Pediatría, Hematológica y Cuidados Intensivos. Las muestras obtenidas se sometieron a métodos fenotípicos para detectar la producción de carbapenemasas. La identificación bacteriana se realizó utilizando espectrometría de masas. El perfil de sensibilidad a los antimicrobianos se determinó por difusión en disco y la detección de determinantes genéticos se realizó mediante la Reacción en Cadena de la Polimerasa. Resultados: Se observó producción de carbapenemasas en el 34,9\% de los aislamientos. Las especies más frecuentes fueron Klebsiella pneumoniae $(68,4 \%)$ e Escherichia coli $(15,8 \%)$. Se observó una alta tasa de resistencia a múltiples fármacos $(89,5 \%)$ entre los Enterobacterales productores de carbapenemasas (EPC). Se encontró que el $81,6 \%$ de las muestras tenían el gen blakpC y el 15,8\%, blandM. Dos muestras resultaron concomitantemente positivas para los genes blakpc/blandm. Conclusión: Se observó una alta tasa de colonización por EPC multirresistente, con predominio de K. pneumoniae portadora de blakPC.

Palabras clave: Bacterias gramnegativas, Microbiota, Control de infecciones.

\section{INTRODUÇÃO}

A ordem das Enterobacterales inclui várias famílias de bacilos Gram-negativos (BGN) fermentadores de glicose, incluindo a família Enterobacteriaceae ou enterobactérias. Vários desses BGN fazem parte da microbiota intestinal de seres humanos e são importantes patógenos oportunistas, podendo causar tanto infecções de origem comunitária, quanto infecções relacionadas à assistência à saúde (IRAS), tais como infecções respiratórias, urinárias e da corrente sanguínea. Entre as principais espécies e gêneros de importância clínica, frequentemente associadas com IRAS, estão Klebsiella pneumoniae, Escherichia coli, Enterobacter spp., Citrobacter spp., Serratia marcescens, Proteus mirabilis, entre outras (ABBOTT SL, 2011).

Nos Estados Unidos, E. coli e K. pneumoniae foram responsáveis por cerca de $15 \%$ e $8 \%$ das IRAS em geral, estando em $1^{\circ}$ e $3^{\circ}$ lugares, respectivamente, entre os patógenos mais prevalentes associados com IRAS, durante o período de 2011 a 2014 (WEINER LM, et al., 2016). Ainda nesse estudo, a espécie E. coli foi classificada como $01^{\circ}$ agente etiológico de infecções urinárias associadas a cateter, enquanto que, $K$. pneumoniae ficou em $3^{\circ}$ e $4^{\circ}$ lugares, como agente de pneumonias associadas à ventilação mecânica (PAV) e infecções primárias da corrente sanguínea (IPCS) associadas a cateter venoso central, respectivamente (WEINER LM, et al., 2016). No Brasil, dados da Agência Nacional de Vigilância Sanitária (ANVISA), do ano de 2016, apontaram K. pneumoniae como a $2^{\circ}$ causa mais frequente de IPCS, tanto na Unidade de Terapia Intensiva (UTI) adulto, quanto na UTI neonatal (BRASIL, 2017).

Nas últimas décadas, essas bactérias, particularmente $K$. pneumoniae, também têm se destacado pela sua crescente resistência aos principais antimicrobianos utilizados na clínica médica, entre eles, as cefalosporinas de $3^{\text {a }}$ e $4^{\text {a }}$ gerações e, mais recentemente, os carbapenêmicos, considerados drogas de escolha para tratamento de infecções graves por BGN (CODJOE FS e DONKOR ES, 2018; KAYE KS e POGUE JM, 2015). Em 2017, a Organização Mundial de Saúde (OMS) publicou uma lista de patógenos multirresistentes, considerados prioritários para a pesquisa e o desenvolvimento de novos antimicrobianos, 
sendo que as Enterobacterales resistentes a carbapenêmicos e/ou produtoras de beta-lactamases de espectro estendido (Extended-Spectrum Beta-Lactamase, ESBL) figuraram entre os três patógenos classificados na categoria de nível crítico (WILLYARD C, 2017).

A resistência aos carbapenêmicos pode ser devida a vários mecanismos, sendo que, em Enterobacterales o principal deles é a produção de enzimas beta-lactamases, denominadas de carbapenemases, que conferem resistência não só aos carbapenêmicos, mas à maioria dos $\beta$-lactâmicos, incluindo as cefalosporinas (CODJOE FS e DONKOR ES, 2018; GIEDRAITIENÊ A, et al., 2011; PATEL G e BONOMO RA, 2013). As carbapenemases pertencem a três classes moleculares, segundo a classificação de Ambler: A, B e D. As enzimas das classes $A$ e $D$ pertencem ao grupo das serino-beta-lactamases, por apresentarem um resíduo de serina em seus sítios ativos e as da classe $B$, ao grupo das metalo-beta-lactamases (M $\beta L$ ), por requererem íons de zinco para sua atividade hidrolítica. Devido a essa exigência de cátions, as $M \beta L$ são inibidas por agentes quelantes, como o ácido etilenodiaminotetracético (EDTA) (BONOMO RA, 2018).

Entre as carbapenemases da classe A, incluem-se as enzimas Guiana Extended-spectrum (GES) e Klebsiella pneumoniae Carbapenemase (KPC), entre outras. Entre as M $\beta \mathrm{L}$, podemos citar as dos tipos Imipenemase (IMP), Verona Imipenemase (VIM), São Paulo Metalo-beta-lactamase (SPM) e, a mais recentemente descrita, New Delhi Metallo-beta-lactamase (NDM). Já entre as carbapenemases da classe D, também conhecidas como oxacilinases (OXA), destacam-se a OXA-23, OXA-24, OXA-58 e OXA-143, comumente descritas em Acinetobacter spp., e a OXA-48, que tem sido relatada entre amostras de enterobactérias (BONOMO RA, 2018).

Atualmente, as carbapenemases das classes A, B e D de maior importância epidemiológica e clínica entre as Enterobacterales são as dos tipos KPC, NDM e a OXA-48, respectivamente, sendo que os determinantes genéticos dessas enzimas estão localizados em elementos genéticos móveis, como plasmídeos e transposons, aumentando sua transmissibilidade horizontal e disseminação (NORDMANN P e POIREL L, 2014). Adicionalmente, esses elementos móveis frequentemente carreiam simultaneamente genes de resistência a outras classes de antimicrobianos, como aminoglicosídeos e quinolonas, resultando em um fenótipo de multirresistência (PATEL G e BONOMO RA, 2013).

Pacientes hospitalizados e colonizados por Enterobacterales produtoras de carbapenemases (EPC) são reservatórios silenciosos dessas bactérias e, além de apresentarem maior risco de adquirirem infecções extraintestinais subsequentes, podem atuar como fontes de infecções para outros pacientes, bem como de surtos hospitalares (MARTIN RM, et al., 2016). A detecção dessas amostras e o rastreamento desses pacientes é uma medida importante para o controle da disseminação desses microrganismos no ambiente hospitalar, bem como de IRAS causadas por bactérias carbapenemase positivas (ADLER A, et al., 2011; ENFIELD KB, et al., 2014; VIAU R, et al., 2016).

Nesse contexto, o objetivo desse trabalho foi detectar fenotípica e genotipicamente amostras de Enterobacterales produtoras de carbapenemases em pacientes colonizados e internados em um hospital universitário da região metropolitana do Rio de Janeiro, bem como verificar a distribuição de espécies entre essas amostras e seus perfis de sensibilidade.

\section{MÉTODOS}

No período de setembro a outubro de 2018, foi isolado um total de 109 amostras obtidas de culturas de vigilância em meio CHROMagar ESBL (Plastlabor, Rio de Janeiro, RJ), durante a Rotina do Laboratório de Microbiologia, do Serviço de Patologia de um Hospital Universitário, de alta complexidade, da região metropolitana do Rio de Janeiro, a partir de swabs retais provenientes de 57 pacientes internados em doze setores hospitalares.

Os swabs foram coletados de todos pacientes admitidos e depois, semanalmente, naqueles pacientes internados nos seguintes setores: Emergência, Centro de Diálise, Pediatria, Unidade Coronariana, Unidade Hematológica, UTI adulto e UTI neonatal.

A UTI adulto foi a unidade com maior isolamento de amostras $(n=24 ; 22 \%)$, seguida da Unidade Coronariana $(n=18 ; 16,5 \%)$ e da Unidade Hematológica $(n=15 ; 13,8 \%)$. As amostras isoladas que 
apresentaram crescimento no CHROMagar ESBL sugestivo dos gêneros Klebsiella spp., Enterobacter spp. e Citrobacter spp. (colônias azuis esverdeadas) e da espécie Escherichia coli (colônias rosadas) foram selecionadas.

As 109 amostras isoladas foram submetidas ao Método de Inativação do Carbapenêmico Modificado (CIMm) para verificação da produção de carbapenemase. Em adição, todas as amostras também foram submetidas ao Método de Inativação do Carbapenêmico com EDTA (CIMe), para classificação da carbapenemase em serino-beta-lactamase ou metalo-beta-lactamase (MBL). Os resultados dos testes CIMe foram considerados em conjunto com os dos testes CIMm (CLSI, 2018).

Os procedimentos dos testes, bem como a leitura e interpretação dos mesmos foram realizadas de acordo com as recomendações do Clinical and Laboratory Standards Institute (CLSI), em que um aumento $\geq 5 \mathrm{~mm}$ no halo de inibição ao redor do disco de meropenem $(10 \mu \mathrm{g})$, do teste CIMe, quando comparado ao halo de inibição ao redor do disco de meropenem $(10 \mu \mathrm{g})$, do teste $\mathrm{CIMm}$, foi considerado um resultado positivo para produção de MßL (CLSI, 2018).

Amostras pertencentes à coleção bacteriana do laboratório e previamente bem caracterizadas como sendo produtoras de KPC ou de NDM, foram utilizadas como controles serino-beta-lactamase positivo e metalobeta-lactamase positivo, respectivamente.

As amostras positivas para a produção de carbapenemases no teste fenotípico CIMm foram identificadas quanto à espécie por meio da técnica de Espectrometria de Massa com Ionização por Dessorção a Laser Assistida por Matriz e analisador de Tempo de Voo - MALDI-TOF MS (Matrix-Assisted Laser Desorption/lonization-Time of Flight Mass Spectrometry), utilizando a plataforma MALDI Biotyper (Bruker Daltonics, Alemanha).

Os perfis de suscetibilidade das amostras positivas nos testes $\mathrm{CIMm}$ foram verificados por meio do método de difusão em ágar, de acordo com as recomendações e critérios do CLSI, utilizando-se os seguintes discos de antimicrobianos (Cecon, São Paulo, SP): amicacina $(30 \mu \mathrm{g})$, cefotaxima $(30 \mu \mathrm{g})$, ceftriaxona $(30 \mu \mathrm{g})$, cefepima $(30 \mu \mathrm{g})$, ceftazidima $(30 \mu \mathrm{g})$, ciprofloxacina $(5 \mu \mathrm{g})$, ertapenem $(10 \mu \mathrm{g})$, gentamicina $(10 \mu \mathrm{g})$, imipenem $(10 \mu \mathrm{g})$, meropenem $(10 \mu \mathrm{g})$, tetraciclina $(30 \mu \mathrm{g})$ e trimetoprima/sulfametoxazol $(1,25 / 23,75 \mu \mathrm{g})(\mathrm{CLSI}, 2018)$. As cepas E. coli ATCC 25922 e Pseudomonas aeruginosa ATCC 27853 foram utilizadas como controles dos testes. Amostras resistentes a pelo menos um agente de três ou mais classes de antimicrobianos foram consideradas multirresistentes (Multidrug-resistant, MDR) (MAGIORAKOS AP, et al., 2012).

As amostras positivas para produção de carbapenemases nos testes fenotípicos foram submetidas a reações em cadeia da polimerase (PCR) Multiplex, para a detecção dos seguintes determinantes genéticos codificadores de carbapenemases: blakPC, blanDM e blaoxA-48-like (MONTEIRO J, et al., 2012). Cada reação foi preparada contendo $2 \mu \mathrm{l}$ de DNA cromossômico, 6,5 $\mu \mathrm{l}$ de água Milli-Q, 0,5 $\mu \mathrm{L}$ (20 pmoles) de cada iniciador para os genes blakpc e blaoxA-48, $1 \mu \mathrm{L}$ (20 pmoles) de cada iniciador para o gene blandm, e $12,5 \mu l$ do JumpStart ${ }^{\mathrm{TM}}$ REDTaq ${ }^{\circledR}$ ReadyMix ${ }^{\mathrm{TM}}$ Reaction Mix (Sigma-Aldrich, Missouri, EUA), totalizando um volume final de $25 \mu \mathrm{L}$.

As condições de amplificação foram: um ciclo de desnaturação inicial a 94ํㅡ por 5 minutos; 30 ciclos de desnaturação ( $94^{\circ} \mathrm{C}$ por 1 minuto), anelamento ( $50^{\circ} \mathrm{C}$ por 1 minuto) e extensão ( $72^{\circ} \mathrm{C}$ por 1 minuto), com um ciclo de extensão final ( $72^{\circ} \mathrm{C}$ por 5 minutos). As reações de PCR foram repetidas duas vezes e para cada reação foi realizado o isolamento do DNA, por meio de lise térmica. A amplificação do DNA foi visualizada através de eletroforese em gel de agarose (Invitrogen, Califórnia, EUA) a 1,5\% em TBE 0,4X. Amostras caracterizadas previamente por sequenciamento como sendo portadoras dos genes blakPC, blaNDM e blaoXA48-like foram utilizadas como controles positivos.

Todos os dados obtidos foram organizados, tratados e analisados através do programa Microsoft ${ }^{\circledR}$ Excel® 2010.

Esse trabalho foi aprovado pelo Comitê de Ética em Pesquisa, da Faculdade de Medicina, da Universidade Federal Fluminense, sob o número CAAE 95984018.6.0000.5243. 


\section{RESULTADOS}

Do total de 109 amostras isoladas a partir das culturas de vigilância de 57 pacientes, 38 (34,9\%) amostras isoladas de $23(40,4 \%)$ pacientes foram positivas nos testes CIMm. Dessas, sete $(18,4 \%)$ amostras foram positivas nos testes CIMe, caracterizando fenotipicamente a presença de enzimas carbapenemases do tipo $\mathrm{M} \beta \mathrm{L}$; as outras 31 amostras $(81,6 \%)$ restantes obtiveram resultados negativos para estes testes, sugerindo a produção de serino-beta-lactamases.

Entre os doze setores hospitalares, sete (58\%) tiveram pacientes colonizados com amostras positivas nos testes fenotípicos: UTI, Unidade Coronariana, Clínica Hematológica, Emergência, Clínica Médica, Clínica Pediátrica e Setor Pós-operatório. Entre esses, a UTI foi o setor com maior percentual de isolamento (13/38 isolados; 34,2\%), seguido da Clínica Hematológica (6/38; 15,8\%).

A espécie mais frequente entre as amostras positivas nos testes fenotípicos para produção de carbapenemases foi $K$. pneumoniae (26/38; 68,4\%), seguida de E. coli $(6 / 38 ; 15,8 \%)$ e Enterobacter asburiae (2/38; $5,3 \%)$. Amostras de Enterobacter cloacae, Citrobacter farmeri, Klebsiella oxytoca e Providencia rettgeri produtoras de carbapenemases, também foram detectadas (1/38 amostra cada; $2,6 \%$ cada).

As taxas de resistência aos carbapenêmicos testados (ertapenem, meropenem e imipenem) foram de $81,6 \%, 78,9 \%$ e $73,7 \%$, respectivamente. As maiores taxas de resistência observadas foram em relação às cefalosporinas ceftriaxona e cefotaxima $(100 \%)$, seguidas de cefepima $(97,4 \%)$ e os menores percentuais foram frente aos aminoglicosídeos testados, amicacina $(23,7 \%)$ e gentamicina $(34,2 \%)$ (Tabela 1).

Tabela 1 - Perfis de suscetibilidade aos antimicrobianos das 38 amostras de Enterobacterales produtoras de carbapenemases, obtidas de pacientes colonizados. Niterói-RJ, 2018.

\section{Perfis de Suscetibilidade}

\section{Antimicrobiano}

\section{Sensível n (\%) Intermediário n (\%) Resistente $\mathbf{n}(\%)$}

\begin{tabular}{lccc}
\hline Cefotaxima & $0(0)$ & $0(0)$ & $38(100)$ \\
Ceftriaxona & $0(0)$ & $0(0)$ & $38(100)$ \\
Cefepima & $1(2,6)$ & $0(0)$ & $37(97,4)$ \\
Ceftazidima & $0(0)$ & $3(7,9)$ & $35(92,1)$ \\
Ciprofloxacina & $2(5,3)$ & $3(7,9)$ & $33(86,8)$ \\
Sulfametoxazole-trimetoprima & $5(13,1)$ & $0(0)$ & $33(86,8)$ \\
Ertapenem & $3(7,9)$ & $4(10,5)$ & $31(81,6)$ \\
Meropenem & $6(15,8)$ & $2(5,3)$ & $30(78,9)$ \\
Imipenem & $3(7,9)$ & $7(18,4)$ & $28(73,7)$ \\
Tetraciclina & $20(52,6)$ & $0(0)$ & $18(47,4)$ \\
Gentamicina & $20(52,6)$ & $5(13,1)$ & $13(34,2)$ \\
Amicacina & $25(65,8)$ & $4(10,5)$ & $9(23,7)$ \\
\hline
\end{tabular}

Fonte: Lima MMS, et al., 2020. 
O perfil de resistência cefepima / cefotaxima / cefotriaxona / ceftazidima / ertapenem / meropenem / imipenem / ciprofloxacina / sulfametoxazol-trimetoprima foi o mais frequente (11/38; 29\%) e multirresistência foi observada em 34 amostras (89,5\%) (Tabela 2).

Tabela 2 - Perfis de resistência aos antimicrobianos das 38 amostras de Enterobacterales produtoras de carbapenemases, obtidas de pacientes colonizados. Niterói-RJ, 2018.

\begin{tabular}{|c|c|c|}
\hline \multirow{2}{*}{ Perfis de Resistência } & \multicolumn{2}{|c|}{ Amostras Bacterianas } \\
\hline & $\mathbf{n}$ & $\%$ \\
\hline CPM, CTX, CFO, CAZ, ERT, MER, IPM, CIP, SUT* & 11 & 29,0 \\
\hline CPM, CTX, CFO, CAZ, ERT, MER, IPM, GEN, TET, CIP, SUT* & 4 & 10,6 \\
\hline CPM, CTX, CFO, CAZ, ERT, MER, IPM, TET, CIP, SUT* & 3 & 7,9 \\
\hline CPM, CTX, CFO, CAZ, ERT, MER, CIP, SUT* & 3 & 7,9 \\
\hline CPM, CTX, CFO, CAZ, ERT, MER, GEN, TET, CIP, SUT* & 2 & 5,3 \\
\hline CPM, CTX, CFO, CAZ, ERT, MER, IPM, GEN, AMI, CIP, SUT* & 2 & 5,3 \\
\hline CPM, CTX, CFO, CAZ, IPM, GEN, AMI, TET, CIP, SUT* & 2 & 5,3 \\
\hline CPM, CTX, CFO, CAZ, ERT, MER, IPM, GEN, AMI, TET, CIP, SUT* & 1 & 2,6 \\
\hline CPM, CTX, CFO, CAZ, ERT, MER, GEN, AMI, TET, CIP, SUT* & 1 & 2,6 \\
\hline CPM, CTX, CFO, CAZ, ERT, IPM, GEN, TET, CIP, SUT* & 1 & 2,6 \\
\hline CPM, CTX, CFO, CAZ, ERT, MER, IPM, AMI, CIP, SUT* & 1 & 2,6 \\
\hline CPM, CTX, CFO, CAZ, TET, CIP, SUT* & 1 & 2,6 \\
\hline CPM, CTX, CFO, CAZ, TET, CIP* & 1 & 2,6 \\
\hline CPM, CTX, CFO, MER, TET, SUT* & 1 & 2,6 \\
\hline CTX, CFO, ERT, MER, IPM, GEN, AMI & 1 & 2,6 \\
\hline CPM, CTX, CFO, CAZ, IPM, TET & 1 & 2,6 \\
\hline CPM, CTX, CFO, ERT, IPM, AMI & 1 & 2,6 \\
\hline CPM, CTX, CFO, CAZ & 1 & 2,6 \\
\hline Total & 38 & $100 \%$ \\
\hline
\end{tabular}

Legenda: AMI: Amicacina, CAZ: Ceftazidima, CIP: Ciprofloxacina, CPM: Cefepime, CTX: Cefotaxima, CFO: Ceftriaxona, ERT: Ertapenem, GEN: Gentamicina, IPM: Imipenem, MER: Meropenem, TET: Tetraciclina, SUT: Sulfametoxazol-trimetoprima. *Fenótipo de multirresistência (MDR).

Fonte: Lima MMS, et al., 2020.

Cabe ressaltar que todas as amostras positivas nos testes fenotípicos e/ou moleculares apresentaram resistência a pelo menos um dos carbapenêmicos testados, com exceção de três, que foram intermediárias a pelo menos um desses antimicrobianos (CBAC 14, intermediária ao meropenem/blandM positiva; CBAC 175, intermediária ao imipenem/blakPC positiva; CBAC 189, intermediária ao ertapenem e ao imipenem/blakPC positiva).

Entre os determinantes genéticos investigados, o blakpc foi o mais frequente (33/38; $86,8 \%$ ), seguido do bla NDM $_{\text {( }}(6 / 38 ; 15,8 \%)$. O gene blakPC foi observado em várias das espécies de Enterobacterales isoladas, porém a espécie mais associada a esse determinante genético foi $K$. pneumoniae (25/38; $65,8 \%)$. O gene blandM foi observado apenas entre amostras das espécies K. pneumoniae $(\mathrm{n}=2)$, E. coli $(\mathrm{n}=3)$ e $P$. rettgeri $(\mathrm{n}=1)$. O gene blaoxA-48-like não foi detectado em nenhuma amostra e uma amostra $(2,6 \%)$ foi negativa para todos os genes pesquisados. Ressalta-se que em duas amostras (5,3\%) (uma de K. pneumoniae e uma de E. coli) os genes

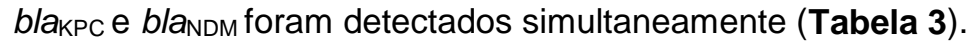


Tabela 3 - Distribuição de espécies de Enterobacterales e presença de determinantes genéticos para produção de carbapenemases entre as 38 amostras produtoras de carbapenemases, de acordo com testes fenotípicos. Niterói-RJ, 2018.

\begin{tabular}{|c|c|c|c|c|c|c|}
\hline \multirow{2}{*}{ Espécie } & \multirow{2}{*}{ Total } & \multicolumn{5}{|c|}{ Determinante genético para carbapenemase, $n(\%)$} \\
\hline & & $b_{\text {bla }}$ & $b / a_{N D M}$ & blaoxA-48-like & bla $a_{\mathrm{KPC}} / \mathrm{bla}_{\mathrm{NDM}}$ & Negativo \\
\hline Klebsiella pneumoniae & 26 & $24(92,3)$ & $1(3,8)$ & $0(0)$ & $1(3,8)$ & $0(0)$ \\
\hline Escherichia coli & 6 & $2(33,3)$ & $2(33,3)$ & $0(0)$ & $1(16,7)$ & $1(16,7)$ \\
\hline Enterobacter asburiae & 2 & $2(100)$ & $0(0)$ & $0(0)$ & $0(0)$ & $0(0)$ \\
\hline Enterobacter cloacae & 1 & $1(100)$ & $0(0)$ & $0(0)$ & $0(0)$ & $0(0)$ \\
\hline Klebsiella oxytoca & 1 & $1(100)$ & $0(0)$ & $0(0)$ & $0(0)$ & $0(0)$ \\
\hline Citrobacter farmeri & 1 & $1(100)$ & $0(0)$ & $0(0)$ & $0(0)$ & $0(0)$ \\
\hline Providencia rettgeri & 1 & $0(0)$ & $1(100)$ & $0(0)$ & $0(0)$ & $0(0)$ \\
\hline Totais & 38 & $31(81,5)$ & $4(10,5)$ & $0(0)$ & $2(5,3)$ & $1(2,6)$ \\
\hline
\end{tabular}

Fonte: Lima MMS, et al., 2020.

Comparando-se os resultados dos testes moleculares com os dos testes fenotípicos, verificou-se que houve concordância de $92,1 \%$ dos resultados (35/38). Uma amostra positiva nos testes $\mathrm{CIMm} / \mathrm{CIMe}$, identificada como sendo produtora de MBL (CBAC 60, E. coli) não apresentou nenhum dos genes investigados. Outras duas amostras CIMe positivas, classificadas como produtoras de MBL (CBAC 26, E. coli e CBAC 189, C. farmeri) apresentaram o gene blakPC e foram negativas para o gene blandM.

As duas amostras que apresentaram os genes blakP/blanDM concomitantemente, foram classificadas como sendo produtoras de serino-beta-lactamases, de acordo com os testes fenotípicos.

\section{DISCUSSÃO}

Esse é o primeiro estudo de investigação laboratorial fenotípica e genotípica de Enterobacterales produtoras de carbapenemases em pacientes colonizados, no hospital em questão. Um elevado percentual de pacientes colonizados por EPC (34,9\%) foi encontrado; um percentual mais baixo $(24,3 \%)$ foi observado em outro estudo com pacientes colonizados, internados em um hospital de Porto Alegre, bem como o encontrado em um estudo realizado na China, com pacientes de UTI colonizados por $K$. pneumoniae resistentes a carbapenêmicos (20,8\%) (PEREZ LRR e DIAS CG, 2016; SHU L, et al., 2019).

O setor hospitalar com maior percentual de pacientes colonizados por EPC foi a UTI. Esse resultado está em concordância com o de outros estudos, que apontam a admissão na UTI como um dos fatores de risco para colonização por EPC (CHIOTOS K, et al., 2017; SCHECHNER V, et al., 2012).

A espécie mais frequente, entre as amostras positivas para produção de carbapenemase, foi $K$. pneumoniae, como já observado em diferentes estudos com pacientes infectados ou colonizados por EPC no mundo, incluindo o Brasil (RODRIGUES PL, et al., 2015; SAMPAIO JLM e GALES AC, 2016; THADEN JT, et al., 2014; ZARAKOLU P, et al., 2016). Gorrie CL et al. (2017), em seu estudo com amostras de colonização obtidas de pacientes internados na UTI de um hospital universitário na Austrália, verificaram uma elevada taxa de colonização por $K$. pneumoniae entre esses pacientes e uma associação significativa entre a colonização por essa bactéria e a posterior aquisição de infecção, sendo uma grande proporção dessas infecções de origem endógena.

As maiores taxas de resistência foram observadas frentes às cefaloporinas de $3^{a}$ e $4^{a}$ gerações, o que já era esperado, uma vez que o meio utilizado foi um meio seletivo para isolamento de amostras produtoras de ESBL, enzimas que conferem resistência a esses antimicrobianos; por outro lado, em geral, as carbapenemases conferem resistência à maioria dos beta-lactâmicos, incluindo não só as cefalosporinas, mas também os carbapenêmicos (WALSH TR, 2010). 
Todas as amostras EPC apresentaram resistência ou foram classificadas como intermediárias a pelo menos um dos três carbapenêmicos testados e um elevado percentual dessas amostras apresentou multirresistência $(89,5 \%)$. Amostras produtoras de KPC ou NDM frequentemente são multirresistentes, o que aumenta o impacto da sua disseminação no ambiente hospitalar (PORRECA AM, et al., 2018; VIVAS R, et al., 2020).

A colonização por EPC é uma fonte de transmissão cruzada e, além disso, pacientes colonizados apresentam risco mais elevado de desenvolverem IRAS por EPC, dependendo de diversos fatores, como a exposição a determinados antimicrobianos (tais como fluoroquinolonas e metronidazol), internação em unidades de UTI, uso de cateter venoso central e diabetes mellitus (SCHECHNER V, et al., 2012). A detecção precoce desses pacientes pode auxiliar, não só no controle de IRAS por EPC multirresistentes, quanto no manejo terapêutico de pacientes colonizados por essas bactérias.

Amostras produtoras de KPC estão amplamente disseminadas em várias partes do mundo, sendo a KPC considerada endêmica no Brasil (MUNOZ-PRICE LS, et al., 2013; SAMPAIO JLM e GALES AC, 2016). Neste estudo, os resultados dos testes moleculares mostraram que o blakPC foi o determinante genético mais frequente e a espécie KPC positiva mais isolada foi $K$. pneumoniae, embora o gene blakpc também tenha sido detectado em outras cinco espécies de Enterobacterales. Vários estudos no mundo e no Brasil mostram resultados semelhantes (MUNOZ-PRICE LS, et al., 2013; PEREIRA PS, et al., 2013; TAVARES CP, et al., 2015).

Por outro lado, o gene bla de Enterobacterales. Vivas R et al. (2020) observaram um percentual bem mais elevado (50\%), em estudo com amostras de Enterobacterales isoladas em um hospital público de Aracaju. Silva IR et al. (2019) caracterizaram 81 amostras produtoras de NDM isoladas a partir de swabs retais e materiais clínicos, em nove estados do Brasil e distribuídas em 11 espécies diferentes de BGN, demonstrando a elevada capacidade de disseminação do gene blandM.

Embora o percentual encontrado no presente estudo tenha sido relativamente baixo e em menor número de espécies, os resultados alertam para a ocorrência de amostras bla NDM no hospital investigado e para a necessidade de medidas de controle visando limitar a sua disseminação. No nosso conhecimento, esse é o primeiro relato de amostras de colonização bla ${ }_{\mathrm{NDM}}$ positivas nesse hospital, reforçando a importância do rastreamento laboratorial dessas amostras. É interessante destacar que uma das amostras NDM positivas foi identificada como $P$. rettgeri, uma vez que a primeira descrição de NDM no Brasil foi relacionada com uma amostra de $P$. rettgeri, isolada no Rio Grande do Sul (CARVALHO-ASSEF APDA, et al., 2013).

A presença de amostras portadoras desses determinantes genéticos em pacientes colonizados alerta para a possível ocorrência de infecções por amostras EPC positivas para os genes blakPC ou blandM, nesse ambiente hospitalar. A ocorrência de NDM entre as amostras EPC traz uma preocupação maior, uma vez que a nova combinação de beta-lactâmico/inibidor de beta-lactamase, ceftazidima-avibactam (CAZ/AVI), liberada recentemente para o tratamento de infecções por bacilos Gram-negativos resistentes a carbapenêmicos e multirresistentes, embora apresente atividade frente às serino-beta-lactamases da classe A (KPC, entre outras) e algumas da classe D (OXA-48), não é eficaz no tratamento de infecções causadas por cepas produtoras de $M \beta L$, como a NDM, diminuindo ainda mais as opções terapêuticas (GUIMARÃES T, et al., 2019; SHIRLEY M, 2018).

Neste estudo, foram isoladas duas amostras EPC carreando blakPC e blandM simultaneamente. Amostras com essa característica, embora não muito frequentes, já foram isoladas no Brasil (PEREIRA PS, et al., 2015; QUILES MG, et al., 2015; VIVAS R, et al., 2020). Uma vez que o avibactam não tem ação hidrolítica em NDM, o tratamento com a nova combinação CAZ/AVI pode ter eficácia limitada em infecções causadas por amostras co-produtoras de KPC e NDM. A ocorrência de isolados de colonização com essa característica reforça a necessidade da contínua realização de culturas de vigilância e a detecção de amostras EPC, considerandose que a colonização é considerada um fator de risco para aquisição de infecções subsequentes e uma fonte potencial de surtos hospitalares (FRENCKEN JF, et al., 2018; MARTIN RM, et al., 2016). 
Os testes fenotípicos e genotípicos apresentaram uma boa correlação, com apenas três discrepâncias entre eles. A amostra positiva nos testes CIM, mas negativa na PCR, pode representar um resultado falso positivo nos testes fenotípicos ou ainda, ser portadora de outro determinante genético, que não foi incluído na investigação genotípica realizada. Outras duas amostras foram consideradas produtoras de $\mathrm{M} \beta \mathrm{L}$, nos testes fenotípicos, mas foram positivas apenas para o gene blakPC, sugerindo resultados falso positivos no teste CIMe. Apesar das discrepâncias encontradas, o método CIMm/CIMe foi satisfatório e pode ser uma boa alternativa para detecção fenotípica de EPC, a partir de culturas de vigilância. Entretanto, uma limitação do nosso estudo para a avaliação do desempenho do método CIM, foi a não realização dos testes moleculares em todas as amostras isoladas.

Outras limitações desse estudo foram o curto período de tempo e o número baixo de pacientes incluídos; entretanto, ainda assim foi possível observar uma taxa de colonização por EPC elevada e a ocorrência de diferentes espécies de Enterobacterales produtoras de carbapenemases dos tipos KPC e/ou NDM, no hospital investigado.

\section{CONCLUSÃO}

Os resultados do presente estudo mostraram um alto percentual de amostras de colonização produtoras de carbapenemases e multirresistentes, em pacientes internados em um hospital da região metropolitana do Rio de Janeiro, sendo K. pneumoniae a espécie mais preponderante entre as Enterobacterales isoladas. A carbapenemase do tipo KPC foi a mais frequente, mas amostras produtoras de NDM também foram encontradas, alertando para a ocorrência desta $M \beta L$, no hospital investigado. A detecção laboratorial de amostras de colonização produtoras de carbapenemases, a partir de culturas de vigilância, pode ajudar a prevenir uma maior disseminação dessas amostras no ambiente hospitalar, permitindo a adoção precoce de medidas, tais como precauções de contato e coorte de pacientes colonizados, bem como a limpeza e descontaminação de superfícies, visando ao controle de infecções e de surtos causados por essas bactérias, considerando que EPC frequentemente são multirresistentes e estão associadas com taxas de mortalidade mais elevadas.

\section{REFERÊNCIAS}

1. ABBOTT SL. Klebsiella, Enterobacter, Citrobacter, Serratia, Plesiomonas, and other Enterobacteriaceae. In Versalovic J, Carroll K, Funke G, Jorgensen J, Landry M, Warnock D (ed), Manual of Clinical Microbiology. 10th ed. Washington, DC: ASM Press, 2011; p $639-657$.

2. ADLER A, et al. Laboratory and clinical evaluation of screening agar plates for detection of carbapenem-resistant Enterobacteriaceae from surveillance rectal swabs. Journal of Clinical Microbiology, 2011; 49(6): 2239-2242.

3. BONOMO RA, et al. Carbapenemase-Producing Organisms: A Global Scourge. Clinical Infect Diseases. 2018; 3;66(8):1290-1297.

4. BRASIL, Agência Nacional de Vigilância Sanitária. 2017. Boletim segurança do paciente e qualidade em serviços de saúde $n^{\circ}$ 16: avaliação dos indicadores nacionais das Infecções Relacionadas à Assistência à Saúde (IRAS) e resistência microbiana do ano de 2016 . Disponível https://www20.anvisa.gov.br/segurancadopaciente/index.php/publicacoes/category/boletins-statistico. Acesso em: 02 de dez. 2020.

5. CARVALHO-ASSEF APDA, et al. Isolation of NDM-producing Providencia rettgeri in Brazil. The Journal of Antimicrobial and Chemotherapy, 2013; 68(12): 2956-2957.

6. CHIOTOS $\mathrm{K}$, et al. Multicenter study of the risk factors for colonization or infection with carbapenem-resistant Enterobacteriaceae in children. Antimicrobial Agents and Chemotherapy, 2017; 61(12): e01440-17.

7. CLSI. Performance Standards for Antimicrobial Susceptibility Testing. CLSI supplement M100 (ISBN 1-56238-839-8). 28th ed. Wayne, PA: Clinical and Laboratory Standards Institute, 2018; 257p.

8. CODJOE FS, DONKOR ES. Carbapenem Resistance: A Review. Medical Science, 2018; 6(1):1-28.

9. ENFIELD KB, et al. Control of Simultaneous Outbreaks of Carbapenemase-Producing Enterobacteriaceae and Extensively Drug-Resistant Acinetobacter baumannii Infection in an Intensive Care Unit using interventions promoted in the Centers for Disease Control and Prevention 2012 carbapenemase-resistant Enterobacteriaceae Toolkit. Infection Control and Hospital Epidemiology, 2014; 35(7):810-817.

10. FRENCKEN JF, et al. Associations between enteral colonization with gram-negative bacteria and intensive care unitacquired infections and colonization of the respiratory tract. Clinical Infectious Diseases, 2018; 66(4): 497-503.

11. GIEDRAITIENÊ A, et al. Antibiotic Resistance Mechanisms of Clinically Important Bacteria. Medicina (B Aires), 2011 ; 47(3):137-146. 
12. GORRIE CL, et al. Gastrointestinal Carriage Is a Major Reservoir of Klebsiella pneumoniae Infection in Intensive Care Patients. Clinical Infectious Diseases, 2017; 65(2):208-215.

13. GUIMARÃES T, et al. Ceftazidime-avibactam as salvage therapy for infections caused by Enterobacterales coresistant to carbapenems and polymyxins. Antimicrobial Agents and Chemotherapy, 2019; 63:e00528-19.

14. KAYE KS, POGUE JM. Infections Caused by Resistant Gram-Negative Bacteria: Epidemiology and Management. Pharmacotherapy. 2015; 35(10):949-962.

15. MAGIORAKOS AP, et al. Multidrug-resistant, extensively drug-resistant and pandrug-resistant bacteria: an international expert proposal for interim standard definitions for acquired resistance. Clinical Microbiology and Infection, 2012;18(3):268-281.

16. MARTIN RM, et al. Molecular Epidemiology of Colonizing and Infecting Isolates of Klebsiella pneumoniae. mSphere, 2016; 1(5): e00261-16.

17. MONTEIRO J, et al. Rapid detection of carbapenemase genes by multiplex real-time PCR. Journal of Antimicrobial Chemotherapy, 2012; 67(4): 906-909.

18. MUNOZ-PRICE LS, et al. Clinical epidemiology of the global expansion of Klebsiella pneumoniae carbapenemases. Lancet Infectious Diseases, 2013; 13(9): 785-796.

19. NORDMANN P, POIREL L. The difficult-to-control spread of carbapenemase producers among Enterobacteriaceae worldwide. Clinical Microbiology and Infection, 2014; 20: 821-830.

20. PATEL G, BONOMO RA. "Stormy waters ahead": global emergence of carbapenemases. Frontiers in Microbiology, 2013; 4:48

21. PEREIRA PS, et al. Update of the molecular epidemiology of KPC-2-producing Klebsiella pneumoniae in Brazil: spread of clonal complex 11(ST11, ST437 and ST340). Journal of Antimicrobial Chemotherapy, 2013; 68: 312-316.

22. PEREIRA PS, et al. Coproduction of NDM-1 and KPC-2 in Enterobacter hormaechei from Brazil. Microbial Drug Resistance. 2015; 21(2): 234-236.

23. PEREZ LRR, DIAS CG. Emergence of infections due to a polymyxin resistant KPC-2-producing Klebsiella pneumoniae in critically ill patients: what is the role of a previous colonization? Infection Control and Hospital Epidemiology, 2016; $37(2): 240-241$.

24. PORRECA AM, et al. The Epidemiology, evolution, and treatment of KPC-producing organisms. Current Infectious Disease Reports, 2018; 20:13.

25. QUILES MG, et al. Unusual association of NDM-1 with KPC-2 and armA among Brazilian Enterobacteriaceae isolates. Brazilian Journal of Medical and Biologycal Research, 2015; 48:174-177.

26. RODRIGUES PL, et al. Incidência de Infecções por enterobactérias resistentes a carbapenêmicos em pacientes previamente colonizados. Clinical and Biomedical Research, 2015; 35(3):134-140.

27. SAMPAIO JLM, GALES AC. Antimicrobial resistance in in Brazil: focus on beta-lactams and polymyxins. Brazilian Journal of Microbiology, 2016; 47: 31-37.

28. SCHECHNER V, et al. Asymptomatic rectal carriage of blaKPC producing carbapenem-resistant Enterobacteriaceae: who is prone to become clinically infected? Clinical Microbiology and Infection, 2012; 19: 451-456.

29. SHU L, et al. Prevalence and phenotypic characterization of carbapenem-resistant Klebsiella pneumoniae strains recovered from sputum and fecal samples of ICU patients in Zhejiang Province, China. Infection and Drug Resistance, 2019; 12: 11-18.

30. SHIRLEY M. Ceftazidime-Avibactam: A review in the treatment of serious gram-negative bacterial infections. Drugs, 2018; (78): 675-692.

31. SILVA IR, et al. Distribution of clinical NDM-1-producing gram-negative bacteria in Brazil. Microbial Drug Resistance, 2019: 25(3): 394-399.

32. TAVARES CP, et al. Molecular epidemiology of KPC-2-producing Enterobacteriaceae (non-Klebsiella pneumoniae) isolated from Brazil. Diagnostic Microbiology and Infectious Disease, 2015; 82(4): 326-30.

33. THADEN JT, et al. Rising rates of carbapenem-resistant Enterobacteriaceae in community hospitals: a mixed-methods review of epidemiology and microbiology practices in a network of community hospitals in the southeastern United States. Infection Control and Hospital Epidemiology, 2014; 35(8): 978-983.

34. VIAU R, et al. Intestinal carriage of carbapenemase-producing organisms: current status of surveillance methods. Clinical Microbiology Infections, 2016; 29(1):1-27.

35. VIVAS R, et al. Prevalence of Klebsiella pneumoniae carbapenemase-and New Delhi metallo-beta- lactamase-positive K. pneumoniae in Sergipe, Brazil, and combination therapy as a potential treatment option. Revista da Sociedade Brasileira de Medicina Tropical, 2020; 53:e20200064.

36. WALSH TR. Emerging carbapenemases: a global perspective. International Jornal of Antimicrobrial Agents, 2010; 36S(3): 8-14.

37. WEINER LM, et al. Antimicrobial-Resistant Pathogens Associated With Healthcare-Associated Infections: Summary of Data Reported to the National Healthcare Safety Network at the Centers for Disease Control and Prevention, 20112014. Infection Control and Hospital Epidemiology, 2016;37(11):1288-1301.

38. WILLYARD C. The drug-resistant bacteria that pose the greatest health threats. Nature, 2017; 543(7643):15.

39. ZARAKOLU P, et al. Epidemiology of carbapenem-resistant Klebsiella pneumoniae colonization: a surveillance study at a Turkish university hospital from 2009 to 2013. Diagnostic Microbiology and Infectious Disease, 2016 ; 85 (4): 466470. 